\title{
REFLEXOS DO PROCESSAMENTO DE CANA-DE-AÇÚCAR BISADA NA QUALIDADE DO AÇÚCAR VHP
}

\author{
GUSTAVO HENRIQUE GRAVATIM COSTA* \\ IGOR DOS SANTOS MASSON** \\ JULIANA PELEGRINI ROVIERO* \\ MÁRCIA JUSTINO ROSSINI MUTTON***
}

\begin{abstract}
O presente trabalho teve por objetivo comparar a qualidade do caldo clarificado e do açúcar Very High Purity (VHP), produzido de cana bisada e com cultivar de maturação precoce no início da safra. Instalou-se o experimento na Usina São Martinho S/A, Pradópolis (SP), sendo os processos de clarificação e de produção de açúcar desenvolvidos no Laboratório de Tecnologia do Açúcar e do Álcool, da Faculdade de Ciências Agrárias e Veterinárias (FCAV) da Universidade Estadual Paulista (UNESP), Jaboticabal (SP). Adotouse delineamento experimental inteiramente casualizado, sendo empregadas as cultivares SP83-2847 (bisada) e RB855156 (não bisada), com doze repetições para análises tecnológicas e quatro repetições para a produção de açúcar. O processo de clarificação e o caldo clarificado foram avaliados quanto à velocidade de sedimentação, volume de lodo formado, teor de sólidos solúveis totais, $\mathrm{pH}$, turbidez, compostos fenólicos, cor e amido. Caracterizouse o açúcar quanto aos parâmetros porcentagem de sacarose aparente, cor, compostos fenólicos, amido, filtrabilidade, umidade e fator de segurança. Verificou-se que o processo de clarificação do caldo não foi afetado pelo processamento de cana bisada. No entanto, o caldo clarificado da cultivar SP83-2847 apresentou valor mais elevado para o parâmetro cor e maior teor de sólidos solúveis totais. O açúcar produzido a partir de cana bisada apresentou qualidade similar ao da cultivar de maturação precoce. Conclui-se que o processamento de cana bisada em início de safra não afeta a clarificação do caldo e resulta em açúcar VHP com qualidade similar ao da cultivar de maturação precoce.
\end{abstract}

PALAVRAS-CHAVE: CANA-DE-AÇÚCAR; VHP; CANA BISADA, Saccharum spp.; DEFECAÇÃO SIMPLES.

* Doutorandos em Microbiologia Agropecuária, Departamento de Tecnologia, Faculdade de Ciências Agrárias e Veterinárias (FCAV), Universidade Estadual Paulista (UNESP), Jaboticabal, SP, Brasil (e-mail: ghg_costa@hotmail.com; juroviero@hotmail.com).

** Mestre em Microbiologia Agropecuária, Departamento de Tecnologia, FCAV, UNESP, Jaboticabal, SP, Brasil (e-mail: igor_smasson@yahoo.com.br).

*** Doutora em Agronomia (Solos e Nutrição de Plantas), Professora Adjunta, Departamento de Tecnologia, FCAV, UNESP, Jaboticabal, SP, Brasil (e-mail: mjrmut@fcav.unesp.br). 


\section{INTRODUÇÃO}

O Brasil tornou-se o maior produtor e exportador mundial de açúcar fabricado a partir da cana-de-açúcar. O cultivo da cana continua em crescente expansão em razão do seu direcionamento, tanto para a fabricação de açúcar como de etanol. Como consequência de chuvas irregulares, políticas econômicas ou até do planejamento das empresas, o corte da cana pode ser adiado para a próxima safra.

O excedente na safra 2007/2008 de cana alcançou $4 \%$ (28 milhões de toneladas) (CONAB, 2008). Já na safra 2008/2009, devido à crise mundial, 20 milhões de toneladas de cana deixaram de serem colhidas (CONAB, 2009). Na safra 2009/2010 ocorreram chuvas irregulares no segundo semestre e 50 milhões de toneladas de cana-de-açúcar permaneceram no campo (CONAB, 2010). Entretanto nos anos de 2010, 2011 e 2012 não houve cana excedente, decorrente da seca intensa que atingiu o Brasil.

As canas que permanecem dois verões no campo recebem o nome de cana "bisada". Para algumas usinas tal matéria-prima é considerada apta ao processamento no início de safra devido ao seu alto teor de sacarose e de fibra. No entando, maior período de cultivo pode ter reflexos negativos sobre a qualidade da matéria-prima, uma vez que a cana estará mais sujeita ao ataque de pragas e doenças, além de intempéries climáticas que resultam no tombamento da planta. Como consequência da maior pressão de estressores bióticos e abióticos, o caldo pode apresentar alterações na sua composição químico-tecnológica como, por exemplo, elevadas concentrações de compostos fenólicos e amido (CAROLO, 2009).

A elevada concentração de compostos fenólicos na matéria-prima (tais como tanino, antocianina, sacaretina, melanoidina, xantofila e clorofila, entre outros) pode favorecer a produção de açúcar com coloração acima das especificações (SIMIONI et al., 2006). Esses compostos são solúveis no caldo de cana e reagem em contato com o oxigênio presente no ar e íons de ferro, resultando em compostos com cor escura, especialmente quando o meio apresenta características alcalinas (REIN, 2012).

A presença de amido e dextrana no caldo da cana dificulta a cristalização da sacarose, pois essas biomoléculas além de elevarem a viscosidade da massa cozida interferem negativamente sobre a deposição desses carboidratos na superfície dos cristais. Por outro lado, esses carboidratos podem permanecer nos grãos de açúcar, influenciando negativamente os produtos das indústrias de alimentos, de bebidas e de fármacos (OLIVEIRA, ESQUIAVETO e SILVA JUNIOR, 2007).

As características de conformação do produto final afetam diretamente o preço pelo qual o açúcar será comercializado. Quanto menor a qualidade apresentada pelo açúcar, menor será seu valor comercial em virtude das exigências do mercado nacional e internacional (ALBUQUERQUE, 2011). Dentre os tipos de açúcar comercializados destaca-se o Very High Purity (VHP), que além de ser o mais produzido no Brasil está direcionado para o mercado externo. O açúcar VHP caracterizase por apresentar elevada quantidade de sacarose no cristal (Pol), assim como cor acima de 700 U.I. (Unidades ICUMSA).

Objetivou-se comparar a qualidade de duas cultivares de cana-de-açúcar, sendo uma de maturação precoce e a outra bisada, quanto aos impactos observados na clarificação do caldo e açúcar VHP produzido em início de safra.

\section{MATERIAL E MÉTODOS}

Conduziu-se o experimento em áreas de cultivo de cana-de-açúcar da Usina São Martinho S/A, situada em Pradópolis (SP), no período de março a junho de 2010, utilizando as cultivares RB855156 (cana soca) e SP83-2847 (cana bisada), ambas colhidas mecanicamente.

Adotou-se delineamento experimental inteiramente casualizado, com os tratamentos consistindo de duas cultivares de cana-de-açúcar, sendo uma não bisada de maturação precoce (RB855156) e a outra bisada (SP83-2847). Foram empregadas doze repetições para a clarificação 
do caldo e quatro para a produção de açúcar (BANZATO e KRONKA, 2007).

Da colheita do talhão da cultivar em estudo, doze caminhões selecionados ao acaso foram encaminhados ao Laboratório de Pagamento de Cana pelo Teor de Sacarose (PCTS) da Usina São Martinho S/A. Amostras de 10 -15 kg de cada carga foram desfibradas e o caldo extraído em prensa hidráulica (TANIMOTO, 1964), compondo três amostras por talhão. Ao total, quatro diferentes áreas de cultivo foram estudadas a fim de melhor representar a diferença entre cultivares.

Os caldos foram encaminhados para o Laboratório de Tecnologia do Açúcar e do Álcool, da Faculdade de Ciências Agrárias (FCAV), Universidade Estadual Paulista (UNESP), e submetidos ao processo de clarificação por defecação simples. Para realização desse processo, corrigiu-se o $\mathrm{pH}$ dos caldos até a neutralidade mediante adição de hidróxido de cálcio ( $6{ }^{\circ}$ Bé) aquecendo-os até a ebulição. Os caldos aquecidos foram dispostos em provetas de $1 \mathrm{~L}$ em sistema de decantação aquecido por lâmpadas, com adição prévia de auxiliar de sedimentação (FLOMEX 9034 - 2 mg/ $\mathrm{dm}^{3}$ ), no qual permaneceram por 20 minutos (CTC, 2005).

Determinou-se nesse processo, a velocidade de sedimentação das impurezas $(\mathrm{cm} / \mathrm{min}) \mathrm{e}$ o volume de lodo formado $(\mathrm{mL})(\mathrm{CTC}, 2005)$.

O caldo clarificado foi submetido às seguintes análises: sólidos solúveis totais (CTC, 2005); pH (método potenciométrico) (CTC, 2005); turbidez (expressa em porcentagem, com leitura da transmitância em espectrofotômetro a 620 nm) (COPERSUCAR, 2001); compostos fenólicos totais (FOLIN e CIOCALTEAU, 1927) e amido (CHAVAN, KUMAR e JADHAV, 1991).

Após a clarificação, as três amostras obtidas para cada talhão foram agrupadas, homogeneizadas e concentradas até $60^{\circ}$ Brix em evaporador rotativo de simples efeito. Os 4 xaropes originados por cultivar foram submetidos ao processo de cozimento. Obteve-se a massa cozida em cozedor piloto, à temperatura de $61 \pm 2{ }^{\circ} \mathrm{C}$. Procedeu-se à nucleação de cristais utilizando "sementes" e manteve-se o processo na zona metaestável de supersaturação, mediante alimentação com xarope a $60^{\circ}$ Brix.

A separação do açúcar e do mel ocorreu em centrífuga a $6300 \mathrm{~g}$ por 5 minutos. Utilizou-se vapor para lavagem do açúcar com pressão de $1 \mathrm{kgf} / \mathrm{cm}^{2}$ por 2 segundos no início da centrifugação. $\mathrm{O}$ açúcar centrifugado foi submetido ao processo de secagem, iniciado em secador de fluxo de ar quente e depois em estufa a $35^{\circ} \mathrm{C}$ por $12 \mathrm{~h}$.

Realizaram as seguintes análises no açúcar obtido: compostos fenólicos totais (FOLIN e CIOCALTEAU, 1927); amido (CHAVAN, KUMAR e JADHAV, 1991); cor, filtrabilidade, porcentagem de sacarose aparente (Pol) e umidade (CTC, 2005). Calculou-se também o fator de segurança, segundo metodologia descrita por Hugot (1969).

Os resultados obtidos para as avaliações realizadas no caldo clarificado e no açúcar foram submetidos à análise de variância pelo Teste $\mathrm{F}$ e comparação entre médias pelo teste de Tukey (5\%), utilizando-se o Sistema para Análises Estatísticas de Ensaios Agronômicos, AgroEstat (BARBOSA e MALDONADO, 2010).

\section{RESULTADOS E DISCUSSÃO}

As duas cultivares, SP83-2847 e RB855156, apresentavam-se em seu ponto ótimo de maturação e se enquadraram nas especificações recomendadas por Ripoli e Ripoli (2009), com sólidos solúveis totais próximos a $18 \%$, Pol maior que $14 \%$ e pureza acima de $85 \%$. Deve-se ressaltar que as amostras apresentavam teor de compostos fenólicos próximo a $550 \mathrm{mg} / \mathrm{L}$ e cor acima de 15000 Unidades ICUMSA (U.I.). A cultivar bisada revelou $30 \%$ a mais de amido (884 mg/L) que a variedade de maturação precoce (1270 mg/L).

\subsection{CLARIFICAÇÃO DO CALDO}

Comparou-se a dinâmica do processo de clarificação do caldo dos dois genótipos de 
cana-de-açúcar mediante a velocidade de sedimentação e o volume de lodo formado (Tabela 1). A velocidade de sedimentação assume grande importância para a unidade industrial, uma vez que quanto mais rápido os flocos forem para o fundo do decantador, menor o tempo de retenção necessário para a conclusão do processo. Sob esse ponto de vista, o processamento de cana bisada não afetou negativamente a taxa de precipitação das impurezas. Deve-se ressaltar ainda que os valores encontrados mostraram-se similares aos determinados por Madaleno (2006), que avaliando a velocidade de sedimentação da cultivar SP81-3250, observou índices de 3,3 a 3,6 cm/min.

Considerando o volume de lodo sedimentado, resultante da reação entre o cálcio adicionado e os fosfatos de cálcio presentes no caldo, verificou-se que o genótipo de cana-deaçúcar SP83-2847 apresentou quantidade similar de lodo em comparação com a RB855156, ambos da ordem de $25 \%$ do decantador. Tais valores estão próximos aos determinados por Thangamuthu e Khandagave (2010) e Madaleno (2006), os quais trabalhando em sistema de decantação de $1 \mathrm{~L}$ observaram volume de lodo formado após 20 minutos de repouso de 210 a $293 \mathrm{~mL}$.

Após a avaliação do processo de clarificação, os caldos clarificados foram caracterizados quanto ao $\mathrm{pH}$, compostos fenólicos totais, cor, amido, turbidez e sólidos solúveis totais. Constatouse que o processamento de cana bisada resultou em caldo clarificado com $\mathrm{pH}$, fenois totais, amido e turbidez similares ao da variedade de maturação precoce.

Os valores de $\mathrm{pH}(6,4-6,5)$ dos caldos clarificados são ideais para os posteriores processos de produção (concentração e cozimento do xarope), uma vez que em soluções próximas a neutralidade não há degradação da sacarose (ALBUQUERQUE, 2011).

\section{TABELA 1 - VALORES MÉDIOS OBTIDOS PARA A VELOCIDADE DE SEDIMENTAÇÃO, VOLUME DE LODO E pH DO CALDO CLARIFICADO - PRADÓPOLIS (SP) - SAFRA 2010/2011}

\begin{tabular}{cccc}
\hline Cultivares & $\begin{array}{c}\text { Velocidade } \\
\text { Sedimentação }(\mathbf{c m} / \mathbf{m i n})\end{array}$ & $\begin{array}{c}\text { Volume de Lodo } \\
(\mathbf{m L})\end{array}$ & $\begin{array}{c}\text { pH do Caldo } \\
\text { Clarificado }\end{array}$ \\
\hline RB855156 & $3,11 \mathrm{~A}$ & $252,50 \mathrm{~A}$ & $6,4 \mathrm{~A}$ \\
SP83-2847 & $3,06 \mathrm{~A}$ & $259,22 \mathrm{~A}$ & $6,5 \mathrm{~A}$ \\
\hline DMS (5 \%) & 0,46 & 35,92 & 0,23 \\
Teste F & $0,04 \mathrm{~ns}$ & $0,15 \mathrm{~ns}$ & $0,79 \mathrm{~ns}$ \\
CV & 17,72 & 16,58 & 4,29 \\
\hline
\end{tabular}

ns = não significativo; letras maiúsculas comparam as médias na coluna. DMS (5 \%) = Desvio Mínimo Significativo. $\mathrm{CV}=$ Coeficiente de Variação.

Avaliando os teores de compostos fenólicos totais do caldo clarificado, observou-se que o processo de defecação simples removeu quatro vezes os teores dessas biomoléculas presentes no caldo original. Tais resultados estão de acordo com Smith e Paton (1985), os quais afirmaram que a adição correta de ácido fosfórico, leite de cal e polieletrólitos podem remover grande parte desses compostos, sendo clorofila, antocianina, xantofila e caroteno os principais fenois encontrados na cana-de-açúcar.

Associado à eliminação de compostos fenólicos verifica-se significativa redução da cor do caldo após o processo de clarificação, uma vez que a cor apresenta relação direta com tais biomoléculas (ROUPA e ASOKAN, 2008). No entanto, o caldo clarificado da cultivar bisada apresentou valor mais elevado para o parâmetro cor que a cultivar de maturação precoce.

Assim como para o parâmetro cor, o teor de sólidos solúveis totais do caldo clarificado da SP83-2847 mostrou-se maior que o da RB855156 (Tabela 2). 
TABELA 2 - VALORES MÉDIOS OBTIDOS PARA COMPOSTOS FENÓLICOS TOTAIS, AMIDO, COR, TURBIDEZ E SÓLIDOS SOLÚVEIS TOTAIS DO CALDO CLARIFICADO PRADÓPOLIS (SP) - SAFRA 2010/2011

\begin{tabular}{cccccc}
\hline Cultivares & $\begin{array}{c}\text { Fenois Totais } \\
\text { (mg/L) }\end{array}$ & $\begin{array}{c}\text { Amido } \\
\mathbf{( m g / L )}\end{array}$ & $\begin{array}{c}\text { Cor } \\
\mathbf{( U . I . )}\end{array}$ & $\begin{array}{c}\text { Turbidez } \\
\mathbf{( \% )}\end{array}$ & $\begin{array}{c}\text { Sólidos } \\
\text { Solúveis Totais } \\
\text { (\%) }\end{array}$ \\
\hline RB855156 & $181,08 \mathrm{~A}$ & $335,95 \mathrm{~A}$ & $5067 \mathrm{~B}$ & $39,49 \mathrm{~A}$ & $16,1 \mathrm{~B}$ \\
SP83-2847 & $188,89 \mathrm{~A}$ & $335,78 \mathrm{~A}$ & $6367 \mathrm{~A}$ & $45,14 \mathrm{~A}$ & $17,3 \mathrm{~A}$ \\
\hline DMS (5 \%) & 33,86 & 9,43 & 563 & 8,36 & 0,92 \\
Teste F & $0,23 \mathrm{~ns}$ & $0,01 \mathrm{~ns}$ & $22,86^{* *}$ & $1,96 \mathrm{~ns}$ & $6,97^{*}$ \\
CV & 21,61 & 3,32 & 11,65 & 23,32 & 6,51 \\
\hline
\end{tabular}

ns = não significativo; * = significativo ao nível de $5 \%$; ** = significativo ao nível de $1 \%$; letras maiúsculas comparam as médias na coluna. DMS (5\%) = Desvio Mínimo Significativo. CV = Coeficiente de Variação.

\subsection{QUALIDADE DO AÇÚCAR}

Após concentração do caldo clarificado, o xarope foi submetido ao processo de cozimento com posterior centrifugação para separação do açúcar e do mel. Os açúcares produzidos a partir das duas cultivares de cana-de-açúcar foram avaliados quanto à cor, compostos fenólicos totais, amido, filtrabilidade (Tabela 3), Pol, umidade e fator de segurança (Tabela 4). Constatou-se que para todos os parâmetros avaliados, o produto resultante do processamento de cana bisada apresentou qualidade similar ao da cultivar de maturação precoce.

Os valores obtidos para a cor do açúcar mostraram-se mais elevados que os adotados (menores que 400 U.I.) pelas indústrias alimentícias (OLIVEIRA, ESQUIAVETO e SILVA JUNIOR, 2007). Esse resultado pode ser devido à elevada presença de compostos fenólicos inclusos nos cristais, os quais ao reagirem com o oxigênio do ar e íons de ferro desencadeiam a formação de produtos coloridos (REIN, 2012). Deve-se ressaltar ainda, que os valores encontrados para essas biomoléculas foram superiores aos relatados por Simioni et al. (2006), os quais determinaram teores entre 78 a $260 \mathrm{mg} / \mathrm{Kg}$ no açúcar VHP. Outro fator que pode estar relacionado com o escurecimento do açúcar reside na elevada quantidade de moléculas de amido, as quais aumentam a viscosidade do material impedindo que o mel da massa cozida seja totalmente eliminado na centrifugação. Tal fato pode ter contribuído ainda para a elevação do teor de umidade do açúcar (ALBUQUERQUE, 2011).

Embora os valores obtidos para a cor do açúcar tenham sido elevados, ainda foram menores que os relatados por Madaleno (2010). Ao estudar os efeitos da época de colheita da cultivar SP80-1842 sobre a qualidade do açúcar VHP, esse autor verificou que o produto resultante do processamento de matéria-prima imatura apresentou cor de 1928 U.I., enquanto que a colheita no ponto de maturação resultou em açúcar com 1026 U.I. A análise de filtrabilidade do açúcar define o tempo necessário para que o produto seja completamente filtrado pela membrana. O tempo de filtração está diretamente ligado à quantidade de compostos com características viscosas, como amido e dextrana, além de grãos de areia presentes no açúcar (OLIVEIRA, ESQUIAVETO e SILVA JUNIOR, 2007). Apesar de não ter sido observada diferença entre o tempo de filtrabilidade das cultivares, esse parâmetro foi considerado elevado, provavelmente, devido à presença de amido no cristal. 
TABELA 3 - VALORES MÉDIOS OBTIDOS PARA COMPOSTOS FENÓLICOS, AMIDO, COR E FILTRABILIDADE DO AÇÚCAR - PRADÓPOLIS (SP)

SAFRA 2010/2011

\begin{tabular}{ccccc}
\hline Cultivares & $\begin{array}{c}\text { Cor } \\
\text { (U.I.) }\end{array}$ & $\begin{array}{c}\text { Fenol } \\
(\mathbf{m g} / \mathbf{K g})\end{array}$ & $\begin{array}{c}\text { Amido } \\
(\mathbf{m g} / \mathbf{K g})\end{array}$ & $\begin{array}{c}\text { Filtrabilidade } \\
(\mathbf{m L} / \mathbf{m i n})\end{array}$ \\
\hline RB855156 & $1753 \mathrm{~A}$ & $596 \mathrm{~A}$ & $690 \mathrm{~A}$ & $2,33 \mathrm{~A}$ \\
SP83-2847 & $1526 \mathrm{~A}$ & $526 \mathrm{~A}$ & $688 \mathrm{~A}$ & $2,53 \mathrm{~A}$ \\
\hline \hline DMS (5 \%) & 282,95 & 119,88 & 167,42 & 0,83 \\
Teste F & $3,17 \mathrm{~ns}$ & $2,03 \mathrm{~ns}$ & $0,01 \mathrm{~ns}$ & $0,29 \mathrm{~ns}$ \\
CV & 10,04 & 12,34 & 14,03 & 26,59 \\
\hline
\end{tabular}

ns = não significativo; letras maiúsculas comparam as médias na coluna. DMS (5\%) = Desvio Mínimo Significativo. $\mathrm{CV}=$ Coeficiente de Variação.

A porcentagem de sacarose aparente (Pol) obtida para ambos os açúcares foi considerada baixa, enquanto a umidade presente no cristal mostrou-se alta (Tabela 4). Esses problemas são decorrentes do processo e não dos tratamentos, uma vez que podem estar associados à centrifugação deficiente para a remoção completa do mel envolto ao cristal e que não foi totalmente eliminado durante a secagem (ALBUQUERQUE, 2011).

O fator de segurança do açúcar determina a possibilidade de armazenagem do produto por longos períodos. Essa grandeza está diretamente relacionada com a quantidade de sacarose e de umidade presente no cristal. Quantidade elevada de umidade resulta na deterioração microbiológica do açúcar, uma vez que a presença de água é fundamental para o desenvolvimento de bactérias e fungos. Nesse sentido, os resultados obtidos (Tabela 4) enquadraram-se nos padrões estabelecidos por Hugot (1969), que determina 0,20 pontos (razão entre Pol e umidade) como o limite para armazenagem segura de açúcar VHP.

\section{TABELA 4 - VALORES MÉDIOS OBTIDOS PARA POL, UMIDADE E FATOR DE SEGURANÇA DO AÇÚCAR - PRADÓPOLIS (SP) \\ SAFRA 2010/2011}

\begin{tabular}{cccc}
\hline Cultivares & Pol (\%) & Umidade (\%) & Fator de Segurança \\
\hline RB855156 & $94,46 \mathrm{~A}$ & $0,56 \mathrm{~A}$ & $0,19 \mathrm{~A}$ \\
SP83-2847 & $94,92 \mathrm{~A}$ & $0,45 \mathrm{~A}$ & $0,12 \mathrm{~A}$ \\
\hline DMS (5 \%) & 3,85 & 0,20 & 0,07 \\
Teste F & $0,09 \mathrm{~ns}$ & $1,85 \mathrm{~ns}$ & $5,91 \mathrm{~ns}$ \\
CV & 2,35 & 23,48 & 27,00 \\
\hline
\end{tabular}

ns = não significativo; letras maiúsculas comparam as médias na coluna. DMS (5\%) = Desvio Mínimo Significativo. $\mathrm{CV}=$ Coeficiente de Variação.

\section{CONCLUSÃO}

O processamento de cana bisada em início de safra não afeta a clarificação do caldo e resulta em açúcar VHP com qualidade similar à da cultivar de maturação precoce. 


\section{ABSTRACT \\ REFLECTIONS OF THE PROCESSING OF "BISADA" SUGARCANE IN THE QUALITY OF VHP SUGAR}

The aim of this study was to compare the quality of clarified juice and VHP (Very High Purity) sugar produced from "bisada" sugarcane and an early maturation variety, at the beginning of harvest. The experiment was conducted at Usina São Martinho S/A, Pradópolis,SP (Brazil). The process of juice clarification and sugar production was conducted at the Laboratory of Technology of Sugar and Alcohol in the College of Agricultural and Veterinary Sciences at the Universidade Estadual Paulista (FCAV/UNESP), Jaboticabal (SP). The experimental design was randomized, and two varieties were used: SP83-2847 ("bisada" sugarcane) and RB855156 (early maturation), with twelve replications for technological analysis and four replications for sugar production. The clarification process and the clarified juice was evaluated in terms of settling rates, volume of sludge that formed, total soluble solids, $\mathrm{pH}$, turbidity, total phenolic compounds, starch and color. The sugar was characterized regarding the percentage of apparent sugar, color, total phenolic compounds, starch, filterability, humidity and safety factor. It was verified that the processing of the juice clarification was not affected by the processing of the "bisada" sugarcane. However, the clarified juice from the SP83-2847 variety had a more intense color and a higher total soluble solids value. The sugar produced from the "bisada" sugarcane presented similar quality to the early maturation variety. It was concluded that the processing of "bisada" sugarcane at the start of the harvest did not affect the juice clarification and resulted in VHP sugar of a quality similar to that of the early maturation variety.

KEY-WORDS: SUGARCANE; VHP; SUGAR; Saccharum spp.; SIMPLE DEFECATION.

\section{REFERÊNCIAS}

1 ALBUQUeRQUe, F.M. Processo de fabricação do açúcar. 3. ed. Recife: Editora Universitária UFPE, 2011. 449 p.

2 BANZATTO, D.A.; KRONKA, S.N. Experimentação agrícola. 4. ed. Jaboticabal: FUNEP, 2006. 237 p.

3 BARBOSA, J.C.; MALDONADO JR, W. AgroEstat: sistema para análises estatísticas de ensaios agronômicos. Versão 1.0. Jaboticabal: UNESP, 2010.

4 CAROLO, A. Aumento da cana bisada preocupa especialistas. Jornal da Cana, v.187, p. 19, jul.2009.

5 CHAVAN, S.M.; KUMAR A.; JADHAV, S.J. Rapid quantitative analysis of starch in sugarcane juice. International Sugar Journal, v.93, n.107, p.56-59, 1991.

6 Companhia Nacional de Abastecimento (CONAB). $3^{\circ}$ Levantamento cana-de-açúcar safra 2008. Disponível em: <http://www.conab.gov.br >. Acesso em: 14 ago. 2013.

7 Companhia Nacional de Abastecimento (CONAB). $3^{\circ}$ Levantamento cana-de-açúcar safra 2009/2010. Disponível em: <http://www.conab.gov.br >. Acesso em: 14 ago. 2013.

8 Companhia Nacional de Abastecimento (CONAB). $2^{\circ}$ Levantamento cana-de-açúcar safra 2010/2011. Disponível em: <http://www.conab.gov.br >. Acesso em: 14 ago. 2013.

9 Centro de Tecnologia Canavieira (CTC). Manual de métodos de análises para açúcar. Piracicaba: Laboratório de Análises, 2005. CD-ROM.

10 Cooperativa de Produtores de Cana-de-Açúcar, Açúcar e Álcool do Estado de São Paulo (COPERSUCAR). Manual de controle químico da fabricação de açúcar. Piracicaba, 2001.

11 FOLIN, O.; CIOCALTEAU, V. On tyrosine and tryptophane determinations in proteins. The Journal of Biological Chemistry, v.73, n.2, p. 627-50, 1927.

12 HUGOT, E. Manual da engenharia açucareira. São Paulo: Mestre Jou, 1969. v.1.

13 MADALENO, L.L. Cigarrinha-das-raízes na cana-de-açúcar e qualidade do açúcar produzido. 2010. 93 f. Tese (Doutorado em Agronomia - Produção Vegetal) - Faculdade de Ciências Agrárias e Veterinárias, Universidade Estadual Paulista, Jaboticabal, 2010.

14 MADALENO, L.L. Infestação de Mahanarva fimbriolata (STAL, 1854) e controle químico na qualidade da matériaprima e clarificação do caldo de cana. 2006. 49 f. Dissertação (Mestrado em Agronomia - Produção Vegetal) Faculdade de Ciências Agrárias e Veterinárias, Universidade Estadual Paulista, Jaboticabal, 2006.

15 OLIVEIRA, D.T.; ESQUIAVETO, M.M.M.; SILVA JUNIOR, J.F. Impacto dos itens da especificação do açúcar na indústria alimentícia. Ciência e Tecnologia de Alimentos, n.27, p.99-102, ago. 2007. 
REIN, P. Cane sugar engineering. $2^{\text {nd }}$ ed. Berlin: Bartens, 2012. $768 \mathrm{p}$.

17 RIPOLI, T.C.C.; RIPOLI, M.L.C. Biomassa de cana-de-açúcar: colheita, energia e ambiente. 2. ed. Piracicaba: Edição dos Autores, 2009. $333 \mathrm{p}$

18 ROUPA, T.R.; ASOKAN, A. Effect of rind pigments and juice colorants on juice claribility, settling time and mud volume of sugarcane. Sugar Tech., v.10, n.2, p. 109-113, Jun.2008.

19 SIMIONI, K.R.; SILVA, L.F.L.F.; BARBOSA, V.; RÉ, F.E.; BERNADINO, C.P.; LOPES, M.L.; AMORIM, H.V. Efeito da variedade e época de colheita no teor de fenóis totais em cana-de-açúcar. STAB Açúcar, Álcool e Subprodutos, v. 24, p. 36-39, mar.2006.

20 SMITH, P.; PATON, N. Sugarcane flavonoids. Sugar Technology, v.12, p.1117-142, 1985.

21 TANIMOTO, T. The press method of cane analysis. Hawaiian Planter's Record, v.57, p.133-150, 1964.

22 THANGAMUTHU, P.; KHANDAGAVE, R.B. A vegetable clarifying agent for cane juice clarification. In: INTERNATIONAL SOCIETY of SUGAR CANE TECHNOLOGISTS'S CONGRESS, 27., 2010, Vera Cruz, México. Anais... Vera Cruz: ISSCT, 2010. p.1937-1942. 